\section{(A) Check for updates}

Cite this: Nanoscale, 2022, 14, 2475

\title{
Norbornane-based covalent organic frameworks for gas separation $\uparrow$
}

\author{
Sushil Kumar, (D) $\ddagger^{a}$ Mahmoud A. Abdulhamid, (D) $\ddagger^{a}$ A. D. Dinga Wonanke, \\ Matthew A. Addicoat (D) $^{b}$ and Gyorgy Szekely (D) *a
}

Covalent organic frameworks (COFs) have emerged as a new class of crystalline porous materials with distinct structural features, such as uniform pore distribution, tunable architecture, and modifiable skeletons. COFs hold significant promise for application in gas separation because of their high Brunauer-EmmettTeller surface area and narrow pore-size distribution, which enable selective separation. The porosity and separation performance of COFs have been finely tuned by structurally modifying the starting materials. Along this direction, for the first time, we prepared $\mathrm{W}$-shaped diamines by catalytic arene-norbornene annulation (CANAL) and then treated them with trialdehyde (Tp) to synthesize novel $\beta$-ketoenaminelinked norbornane-based COFs, i.e., ND-COF-1 and ND-COF-2, via a solvothermal Schiff-base condensation approach. The pore interior was decorated with methyl groups attached to the norbornane unit of the COF skeleton. Both COFs exhibited high chemical stability in different organic solvents and acidic media. Additionally, they showed high $\mathrm{CO}_{2} / \mathrm{N}_{2}$ selectivity compared with those of previously reported COFs. Moreover, their $\mathrm{CH}_{4} / \mathrm{N}_{2}$ separation efficiency was investigated, and the results revealed that ND-COF-1 is more selective than ND-COF-2, which could be attributed to the less hindered pathway offered to methane gas molecules by the framework pore.

Received 17th November 2021 Accepted 19th January 2022 DOI: $10.1039 / \mathrm{d} 1 \mathrm{nr} 07593 \mathrm{~d}$ rsc.li/nanoscale ranging from a few Angstroms to hundreds of nanometers. In the absence of uniform connectivity among the pores, the polymers ultimately compromise the selective gas transport through their structure. Therefore, researchers have devoted significant efforts to study the structure-property relationship of porous materials and their performance. Structural functionalization can be implemented to control the porosity and boost the separation performance of polymers with intrinsic microporosity. Additionally, altering the type of kinked unit induces notable changes to the overall porosity and gas-separation performance. Thus, developing new kinked structures for porous materials has been the object of research focus recently.

The catalytic arene-norbornene annulation (CANAL) reaction was first reported by Xia and co-workers, who used readily available monomers to develop CANAL ladder polymers with high Brunauer-Emmett-Teller (BET) surface areas and tunable microporosities. ${ }^{3}$ However, the ladder polymers were mechanically fragile and, thus, not suitable for forming free-standing membranes. To overcome the fragility limitation, CANAL polyimides and Tröger's base-based polymers were developed, and these polymers exhibited excellent gas-separation performance. $^{4,5}$ Nonetheless, all porous polymers exhibit amorphous-semicrystalline morphologies, which prevent the control of the pore size and formation of ordered structures. Therefore, the foremost objective of researchers is to enhance
${ }^{a}$ Advanced Membranes and Porous Materials Center, Physical Science and Engineering Division (PSE), King Abdullah University of Science and Technology (KAUST), Thuwal 23955-6900, Saudi Arabia. E-mail: gyorgy.szekely@kaust.edu.sa; Tel: +966128082769; http://www.szekelygroup.com

${ }^{b}$ School of Science and Technology, Nottingham Trent University, Nottingham, UK

$\dagger$ Electronic supplementary information (ESI) available. See DOI: 10.1039/ d1nr07593d

$\$$ Both authors have contributed equally. 
the crystallinity and uniform distribution of pores in the structure of polymers, to increase their gas-pair selectivity, sorption efficiency, and permeability.

Unlike polymers, COFs are well known for their crystalline and porous structures, which are generated through a covalent-bond-linked assembly of organic linkers arranged in an ordered form. ${ }^{6-8}$ Owing to their ordered pore structure, uniform porosity, high crystallinity, high accessible surface area, and tunable pore chemistry, COFs have diverse applications, including in optoelectronics, separation, energy storage, sensing, catalysis, and biomedicine. ${ }^{9}$ Conventionally, COFs are synthesized from commercially available symmetric planar organic precursor materials. These materials allow sufficient stacking between the adjacent COF layers via $\pi-\pi$ interactions, which often results in the formation of welldefined porous and crystalline structures. Therefore, fusing kinked norbornane-based CANAL units with a COF backbone could enhance the separation performance of the resulting porous materials. However, to the best of our knowledge, the development of two-dimensional (2D) COFs using kinked norbornane-based CANAL ligands for gas-separation applications has not yet been reported. ${ }^{6,10,11}$

In this study, two new COFs, ND-COF-1 and ND-COF-2, were prepared by incorporating the kinked norbornane-based CANAL diamine unit into the structures of COFs via a Schiffbase condensation reaction utilizing a solvothermal approach. Furthermore, we investigated the effect of the kinked CANAL unit on the crystallinity and porosity of the resulting COFs. Additionally, the performance and selectivity of the obtained COFs in gas separation were evaluated. Both ND-COFs showed a high affinity toward $\mathrm{CO}_{2}$, and ND-COF-1 showed higher $\mathrm{CH}_{4} /$ $\mathrm{N}_{2}$ selectivity than $\mathbf{N D}-\mathbf{C O F}-2$.

\section{Experimental}

\section{Materials}

The trialdehyde precursor, 1,3,5-triformylphloroglucinol (Tp), was synthesized by following a previously reported procedure. $^{12}$ The materials, 4-bromo-3-methylaniline (97\%), 4-bromo-2,5-dimethylaniline (99\%), palladium(II) acetate (99.9\%), and anhydrous 1,4-dioxane (99.8\%), were purchased from Merck. Caesium carbonate (99\%) was purchased from Alfa Aesar. All reagents were used as received without further purification.

\section{Ligand syntheses}

Two kinked norbornane-based CANAL diamines were prepared by following a previously reported procedure, ${ }^{4}$ using the CANAL reaction. The commercially obtained monomers, i.e., 4-bromo-3-methylaniline and 4-bromo-2,5-dimethylaniline, were reacted with palladium(II) acetate $\left[\mathrm{Pd}(\mathrm{OAc})_{2}, 2 \mathrm{~mol} \%\right]$, triphenylphosphine $\left(\mathrm{PPh}_{3}, 4 \mathrm{~mol} \%\right)$, and caesium carbonate $\left(\mathrm{Cs}_{2} \mathrm{CO}_{3}, 1\right.$ equiv.) in dry 1,4-dioxane heated at $150{ }^{\circ} \mathrm{C}$ for $24 \mathrm{~h}$ to afford pure products in $70 \%-75 \%$ yields. The two bromoaniline monomers with the ortho methyl group were selected to achieve high yields and selectivity for the CANAL ligands. The chemical structures of the obtained diamines were confirmed by proton nuclear magnetic resonance $\left({ }^{1} \mathrm{H} \mathrm{NMR}\right),{ }^{13} \mathrm{C} \mathrm{NMR}$, Fourier-transform infrared (FTIR) spectroscopy, and highresolution mass spectrometry measurements.

\section{COF syntheses}

ND-COF-1 and ND-COF-2 were synthesized in a Pyrex tube via the solvothermal Schiff-base condensation reaction of $\mathbf{T p}$ (63 $\mathrm{mg}, 0.3 \mathrm{mmol}$ ) with the corresponding diamines in the presence of $3 \mathrm{M}$ acetic acid solution $(0.3 \mathrm{~mL})$, using $4 \mathrm{~mL}$ of a $1: 1(\mathrm{v} / \mathrm{v})$ mixture of mesitylene and dioxane as the reaction medium (Fig. 1). Subsequently, three consecutive freezepump-thaw cycles were performed. After sealing under vacuum, the tubes were heated to $120{ }^{\circ} \mathrm{C}$ for $72 \mathrm{~h}$. ND-COF-1 and ND-COF-2 were precipitated as yellow solids, after which they were washed with hot water and hot $\mathrm{N}, \mathrm{N}$-dimethylacetamide (DMAc) to remove the excess unreacted precursors and oligomers. After the final washing with acetone at room temperature, the obtained solids were dried at $90{ }^{\circ} \mathrm{C}$ overnight to afford both COFs as yellow powders.

\section{Characterization}

Powder X-ray diffraction spectra were obtained using a Bruker D8 ADVANCE instrument in the $2 \theta$ range of $2.5^{\circ}-40^{\circ}$ and used to evaluate COF crystallinity. The experiment was carried out using a Quartz holder at a scan rate of $1^{\circ} \mathrm{min}^{-1}$ and a step interval of $0.01^{\circ}$. The stretching bands of the functional groups were obtained from FTIR spectra using a Thermo Scientific Nicolet iS10 spectrometer. Solid-state ${ }^{13} \mathrm{C}$ NMR spectroscopy was performed using the Bruker Avance III $400 \mathrm{MHz}$ equipment. Thermal decomposition of COFs was determined by thermogravimetric analyses (TGA, 209 F1 analyzer Netzsch) under an $\mathrm{N}_{2}$ atmosphere at a ramp rate of $10{ }^{\circ} \mathrm{C} \mathrm{min}^{-1}$, in a range of $30^{\circ} \mathrm{C}-900{ }^{\circ} \mathrm{C}$. X-ray photoelectron spectroscopy (XPS) imaging was performed on a Kratos Axis Ultra DLD electron spectrometer. A Magellan FEI 400 was used for scanning electron microscopy (SEM) measurements. The samples were treated with iridium prior to analysis to avoid surface charging. Nitrogen adsorption analyses were conducted on a Micromeritics ASAP 2420 at $-196{ }^{\circ} \mathrm{C}$. The BET surface areas of ND-COF-1 and ND-COF-2 were calculated. A non-localized density functional theory (NLDFT) model was utilized for the calculations of pore size distribution. $\mathrm{CO}_{2}, \mathrm{~N}_{2}$, and $\mathrm{CH}_{4}$ uptake was measured using a Micromeritics ASAP 2020 at 0 and $25^{\circ} \mathrm{C}$, under atmospheric pressure.

\section{Results and discussion}

The two kinked norbornane-based CANAL diamines, i.e., $\operatorname{CANAL}(\mathrm{Me})_{2}$ and $\mathrm{CANAL}(\mathrm{Me})_{4}$, were prepared via a CANAL reaction. ${ }^{4}$ The resulting diamines exhibited a "W"-shaped structure (syn- and anti-isomers) with exo conformation exclusively. ${ }^{4}$ Two new porous crystalline $\beta$-ketoenamine-linked kinked norbornane COFs, i.e., ND-COF-1 and ND-COF-2, were 


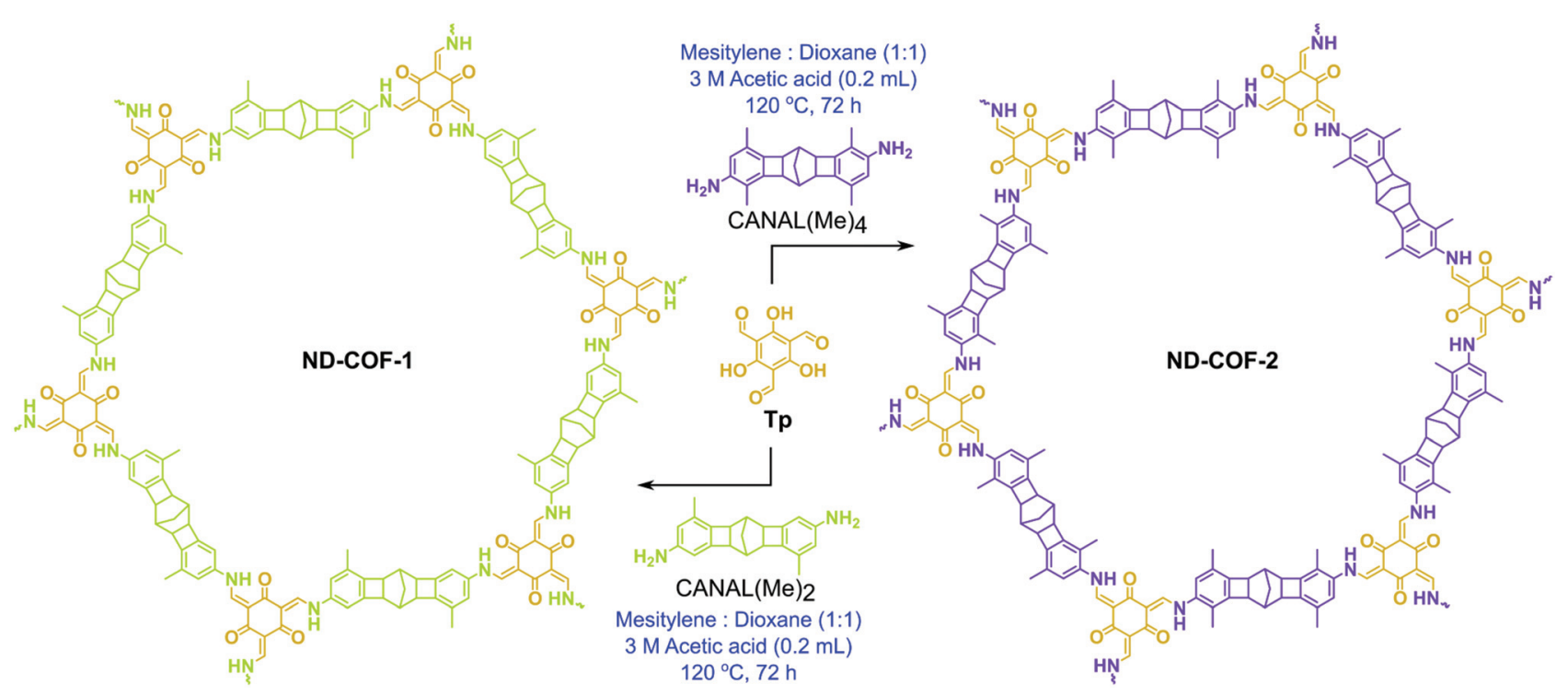

Fig. 1 Solvothermal synthesis of ND-COF-1 and ND-COF-2 via a Schiff-base condensation reaction involving trialdehyde (Tp) and diamines, i.e., CANAL(Me $)_{2}$ and CANAL $(\mathrm{Me})_{4}$, respectively.

synthesized by treating the respective kinked CANAL diamines with Tp using a solvothermal method (Fig. 1). The ND in ND-COF-1 and ND-COF-2 refers to the norbornane-based diamine unit. The PXRD patterns of ND-COF-1 and ND-COF-2 exhibited the first high-intensity peak at $2 \theta=2.5^{\circ}-10^{\circ}$, which corresponds to the diffraction from the [120] plane, whereas a major broad peak appeared at $2 \theta=16^{\circ}-25^{\circ}$, assigned to the diffraction from the [322] plane. Given the three-dimensional nature of the norbornane unit and attached methyl groups, a large distance between the COF layers is expected. In fact, the sensitivity of PXRD in that region is low. During simulation, assigning a $c$ lattice parameter of $12 \AA$, and taking into account the $\mathrm{ABC}$ stacking, gives an inter-linker distance of just below $5 \AA$ (Fig. 2a and b). The relatively high intensity of the first peak indicated the crystalline nature of the ND-COFs. The experimental PXRD patterns matched well with the simulated patterns generated using the $\mathrm{ABC}$ stacking model.

This excellent agreement between the experimental and simulated PXRD patterns was further verified by Pawley refinement (ND-COF-1: $R_{\mathrm{p}}=5.96 \%, R_{\mathrm{wp}}=7.23 \%$ and ND-COF-2: $R_{\mathrm{p}}=$ $\left.6.74 \%, R_{\mathrm{wp}}=7.76 \%\right)$, performed using the Material Studio software refinement package. The FTIR spectra confirmed the absence of any trace of the starting material in ND-COF-1 and ND-COF-2 (Fig. 2c, section S4 of the ESI $\dagger$ ). This was further supported by the disappearance of the $\mathrm{NH}$ stretching band (3178-3200 $\left.\mathrm{cm}^{-1}\right)$ of CANAL(Me $)_{2}$ and $\operatorname{CANAL}(\mathrm{Me})_{4}$ diamines, as well as the $\mathrm{C}=\mathrm{O}$ stretching band $\left(1639 \mathrm{~cm}^{-1}\right)$ of $\mathbf{T p}$. However, the carbonyl $(\mathrm{C}=\mathrm{O})$ peak broadened and merged with the $\mathrm{C}=\mathrm{C}$ stretching band, as shown in Fig. 2c, which is indicative of the extended framework structure. The appearance of the $\mathrm{C}=\mathrm{C}$ stretching band at approx. $1578 \mathrm{~cm}^{-1}$ and a strong band at approx. $1260 \mathrm{~cm}^{-1}$ affirms the formation of $\beta$-ketoenamine linkages between $\mathbf{T p}$ and the respective diamine precursors in the COFs.
The chemical structures of the COFs were determined from the XPS survey profiles (section S5, ESI $\dagger$ ). The XPS full survey profile of both COFs shows three intense peaks, assigned to C (1s), O (1s), and N (1s) signals, respectively. For ND-COF-1, the deconvoluted XPS profile for C 1s showed a signal at 284.27 $\mathrm{eV}$, assigned to the $\mathrm{C}=\mathrm{C}$ moiety of the aromatic ring system. Moreover, the shoulders appearing at 285.13 and $287.21 \mathrm{eV}$ were assigned to the $\mathrm{C}-\mathrm{O}$ and $\mathrm{C}=\mathrm{O}$ bonds, respectively. In addition, a satellite peak was observed at $290.74 \mathrm{eV}$, assigned to the $\pi-\pi^{*}$ transition. The high-resolution spectrum of $\mathrm{N}(1 \mathrm{~s})$ showed one intense signal at $399.52 \mathrm{eV}$, corresponding to the enamine nitrogen of the $\mathrm{C}=\mathrm{C}-\mathrm{NH}-$ bond formed via tautomerism during the condensation reaction. The deconvoluted XPS profile of $\mathrm{O} 1 \mathrm{~s}$ showed a peak at $530.34 \mathrm{eV}$, assigned to the $\mathrm{C}=\mathrm{O}$ group. The shoulder peak appearing at $532.19 \mathrm{eV}$ was assigned to the $\mathrm{C}-\mathrm{O}$ bonds of the framework. For ND-COF-2, the deconvoluted XPS profile of C 1s showed a signal at 284.24 $\mathrm{eV}$, assigned to the $\mathrm{C}=\mathrm{C}$ moiety of the aromatic ring system. The shoulder peaks at 285.48 and $286.35 \mathrm{eV}$ were assigned to the $\mathrm{C}-\mathrm{O}$ and $\mathrm{C}=\mathrm{O}$ bonds of the framework, respectively. In addition, the satellite peak at $290.37 \mathrm{eV}$ was attributed to the $\pi-\pi^{*}$ transition. The N 1s XPS profile showed a signal at 399.53 $\mathrm{eV}$, assigned to the $\mathrm{C}=\mathrm{C}-\mathrm{NH}-$ bond in the framework. The additional signal at $400.87 \mathrm{eV}$ is most probably derived from contamination by another diamine isomer present in small amounts in the COF structure (section 5, ESI $\dagger$ ). The deconvoluted XPS profile of O 1s showed a peak at $530.39 \mathrm{eV}$ (for the $\mathrm{C}=\mathrm{O}$ bond) and a shoulder at $532.0 \mathrm{eV}$, assigned to the $\mathrm{C}-\mathrm{O}$ bonds in the framework.

The formation of the $\beta$-ketoenamine linkage in the framework structure was further verified by solid-state NMR spectroscopy. The ${ }^{13} \mathrm{C}$ solid-state CP-MAS NMR spectra exhibited intense peaks at 184.59 and 184.54 ppm for ND-COF-1 and ND-COF-2, respectively. These peaks can be assigned to the 

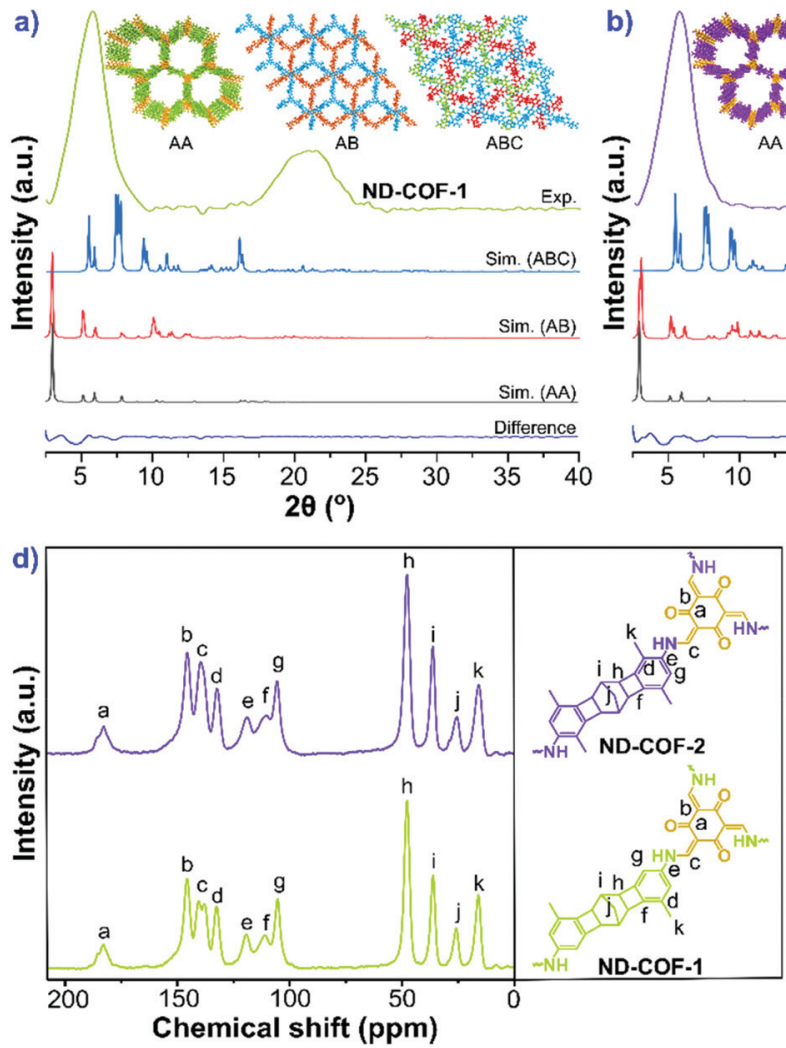
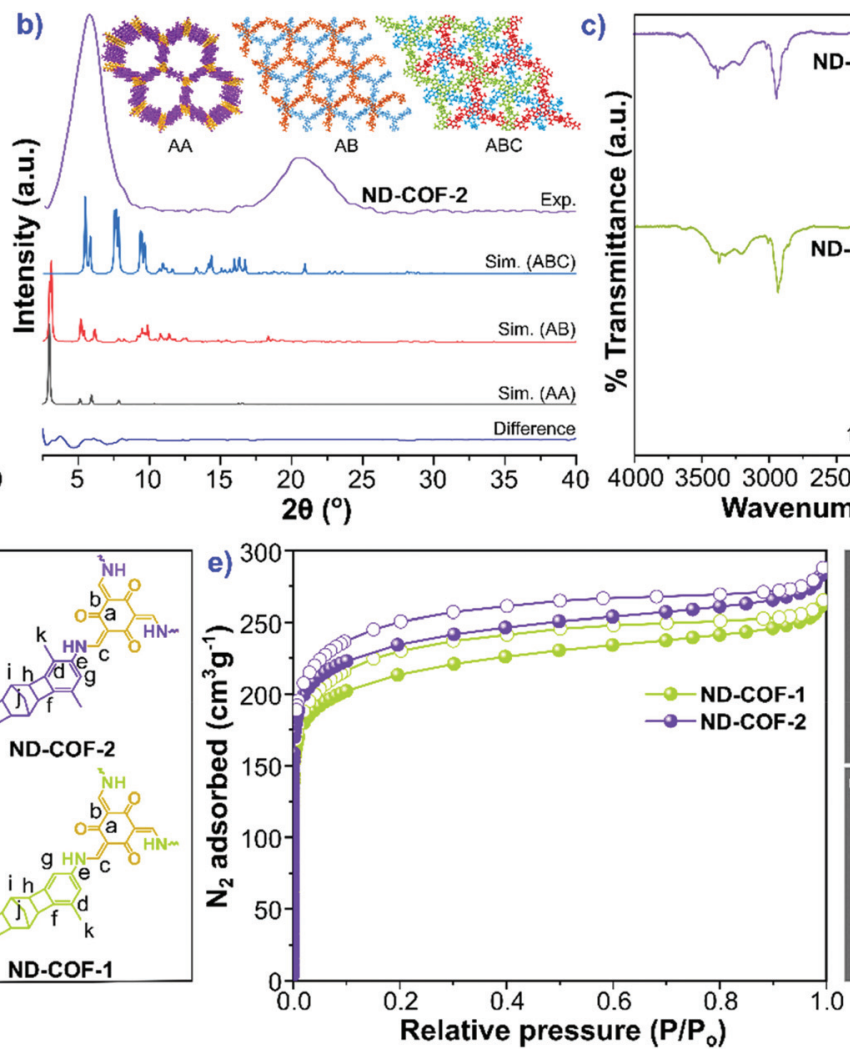
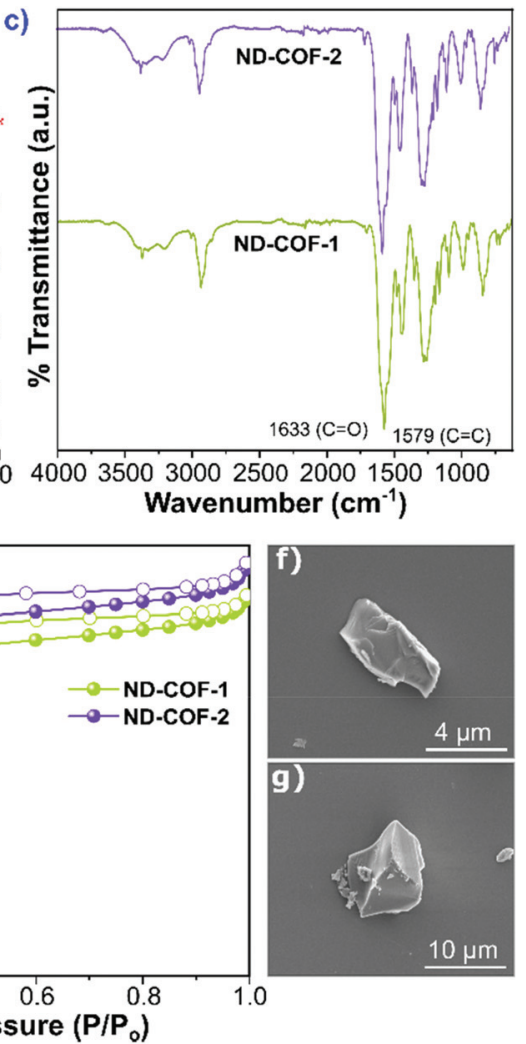

Fig. 2 Experimental and simulated PXRD patterns of (a) ND-COF-1 and (b) ND-COF-2, (c) FTIR spectra of both ND-COFs, (d) solid-state ${ }^{13} \mathrm{C}$ NMR spectra of ND-COF-1 and ND-COF-2, (e) $\mathrm{N}_{2}$ adsorption and desorption isotherms of the ND-COFs recorded at $77 \mathrm{~K}$, and SEM images of (f) ND-COF-1 and (g) ND-COF-2.

carbonyl $(\mathrm{C}=\mathrm{O})$ group of the $\beta$-ketoenamine linkage (Fig. 2d). Conventionally, the carbonyl group $(\mathrm{C}=\mathrm{O})$ of $\mathrm{Tp}$ resonates downfield at $192 \mathrm{ppm} .{ }^{13}$ An upfield shift of the $\mathrm{C}=\mathrm{O}$ peak to $184 \mathrm{ppm}$ for the ND-COFs suggests the formation of a keto group in the framework. In addition, the absence of the characteristic $\mathrm{C}=\mathrm{O}$ peak (assigned to the aldehyde moiety present in Tp) at $192 \mathrm{ppm}$ in the NMR spectra of the ND-COFs suggests the total consumption of the Tp precursor. The NMR peaks resonating in the range of 100-150 ppm can be assigned to the carbon atoms of the aromatic ring. The NMR peaks appearing in the range of $22-55 \mathrm{ppm}$ were assigned to the carbon signals of the norbornane moiety in the framework. The peak resonating at approx. $16 \mathrm{ppm}$ corresponds to the $\mathrm{sp}^{3}$ carbon (methyl group) of the COFs.

The permanent porosities of ND-COF-1 and ND-COF-2 were determined by measuring the nitrogen gas uptake isotherm at $-196{ }^{\circ} \mathrm{C}$ (Fig. 2e). Both COFs exhibited the type-I gas adsorption isotherm, which indicates their microporous nature. The BET surface areas of activated ND-COF-1 and ND-COF-2 were determined to be 794 and $879 \mathrm{~m}^{2} \mathrm{~g}^{-1}$, respectively, which are similar values. However, the calculated surface area using a $1.86 \AA$ radius probe to model $\mathrm{N}_{2}$ is quite sensitive to the position of the methyl groups, because the methyl groups may create triangular pockets in $\mathrm{ABC}$ stacking. Most probably, ND-COF-2 has somewhat better crystallinity, which resulted in the increase in the surface area compared to ND-COF-1. A comparison of theoretical and experimental surface areas calculated for both COFs is given in Table S7, ESI. $\dagger$ Due to the extended length of the organic diamine linkers, the iso-reticulated structures of the ND-COFs exhibited pore apertures lower than $20 \AA$ A. Using the NLDFT method, the pore-size distributions of both ND-COF-1 and ND-COF-2 were calculated to be approx. $16.3 \AA$ and $14.5 \AA$, respectively. The pore size values calculated from the $\mathrm{ABC}$ crystal model were found to be close to the experimental values (Fig. S19 and S20, ESI $\dagger$ ). The SEM images revealed that ND-COF-1 and ND-COF-2 crystallized in a sheet-like morphology (Fig. 2f and g). TGA of activated ND-COF-1 and ND-COF-2 was performed to determine the thermal stability of the framework and the presence of any guest molecule in the pores (section S6, ESI + ). Both COFs showed high thermal stability up to approx. $380{ }^{\circ} \mathrm{C}$. A gradual weight loss after $380{ }^{\circ} \mathrm{C}$ was observed, attributable to the decomposition of the COF structure.

To investigate the chemical stabilities of ND-COF-1 and ND-COF-2 in solvents, they were immersed in different organic solvents, including DMAc, methanol, tetrahydrofuran (THF), hexane, and water, for seven days (section S8, ESI + ). Both COFs were stable in these solvents. The crystallinity of the treated COFs was examined by PXRD (section S8, ESI $\dagger$ ). The absence of significant changes in the relative peak intensities and peak 
positions in the PXRD patterns of the respective COFs indicates their high chemical stability in the examined solvents, including water. A similar observation was made in the FTIR spectra of the treated COFs, where no significant changes in the characteristic FT-IR stretches were observed (section S8, ESI $\dagger$ ).

The stability of ND-COF-1 and ND-COF-2 was further examined in acids (section S8, ESI $\dagger$ ). Both COFs were submerged in $5 \mathrm{~mL}$ of $3 \mathrm{M} \mathrm{HCl}$ solution for seven days. The PXRD patterns of both acid-treated COFs showed no noticeable variation in the relative peak intensities and positions, which indicated the high chemical stability of both COFs. Similarly, no significant variation in the IR stretches was observed, indicating the intactness of the framework even in acidic environments. Furthermore, we evaluated the chemical stability of both COFs under alkaline conditions (section S8, ESI $\dagger$ ). Both COFs were treated with $3 \mathrm{M} \mathrm{NaOH}$ solution for seven days. The PXRD pattern of ND-COF-1 showed no significant variation when compared to that of the pristine COF. Conversely, the PXRD pattern of ND-COF-2 exhibited an additional signal along with the characteristic COF peak, which indicated the disintegration of the framework. Thus, ND-COF-1 is more stable in alkaline media than ND-COF-2. The FTIR spectra of the treated COFs further support this result.

To explore the separation performance of the COFs, we conducted sorption experiments at 1 bar with three different gases: $\mathrm{CO}_{2}, \mathrm{~N}_{2}$, and $\mathrm{CH}_{4}$. The adsorption isotherms of $\mathrm{CO}_{2}, \mathrm{~N}_{2}$, and $\mathrm{CH}_{4}$ measured at $0{ }^{\circ} \mathrm{C}$ and $25^{\circ} \mathrm{C}$ are presented in Fig. 3.
Both ND-COF-1 and ND-COF-2 demonstrated high $\mathrm{CO}_{2}$ adsorption capacities of 2.24 and $2.12 \mathrm{mmol} \mathrm{g}^{-1}$, respectively, at $0{ }^{\circ} \mathrm{C}$ and 1 bar (Fig. 3). Interestingly, ND-COF-1 exhibited similar $\mathrm{CO}_{2}$ and $\mathrm{CH}_{4}$ sorption capacities (Fig. 3a-c), whereas the $\mathrm{CO}_{2}$ sorption capacity of ND-COF-2 was $100 \%$ higher than that for $\mathrm{CH}_{4}$ (Fig. 3d-f). The high $\mathrm{CO}_{2}$ adsorption performance of ND-COF-2 relative to that of ND-COF-1 is attributable to the higher surface area and somewhat better crystallinity of ND-COF-2. Notably, the $\mathrm{N}_{2}$ sorption capacity at room temperature under atmospheric pressure is marginal because of its low critical temperature (low condensability).

The effect of temperature on the COF separation performance was evaluated at $0{ }^{\circ} \mathrm{C}$ and $25{ }^{\circ} \mathrm{C}$ at 1 bar. In particular, a $59 \%$ reduction in the $\mathrm{CO}_{2}$ sorption capacity of ND-COF-1 was observed when the temperature was increased from $0{ }^{\circ} \mathrm{C}$ to $25{ }^{\circ} \mathrm{C}$ (Fig. 3a and b). This corresponded to the change from 2.245 to $1.416 \mathrm{mmol} \mathrm{g}^{-1}$. However, for ND-COF-2, a $48 \%$ reduction in the $\mathrm{CO}_{2}$ sorption capacity was observed, corresponding to the change from 2.124 to $1.428 \mathrm{mmol} \mathrm{g}^{-1}$ (Fig. 3d and e). Under the same conditions, both COFs exhibited high percentage reductions in $\mathrm{N}_{2}$ and $\mathrm{CH}_{4}$ sorption capacities compared to their $\mathrm{CO}_{2}$ sorption capacities. In particular, ND-COF-1 exhibited $59 \%$ and $83 \%$ reductions in $\mathrm{N}_{2}$ and $\mathrm{CH}_{4}$ sorption capacities, respectively, whereas ND-COF-2 showed $275 \%$ and $54 \%$ reductions, respectively. Table 1 shows the $\mathrm{CO}_{2}$ sorption capacities of ND-COF-1 and ND-COF-2 compared with those of the $\mathbf{T}$-based COFs reported in the literature. The obtained $\mathrm{CO}_{2}$ sorption capacities of ND-COF-1 and ND-COF-2 were
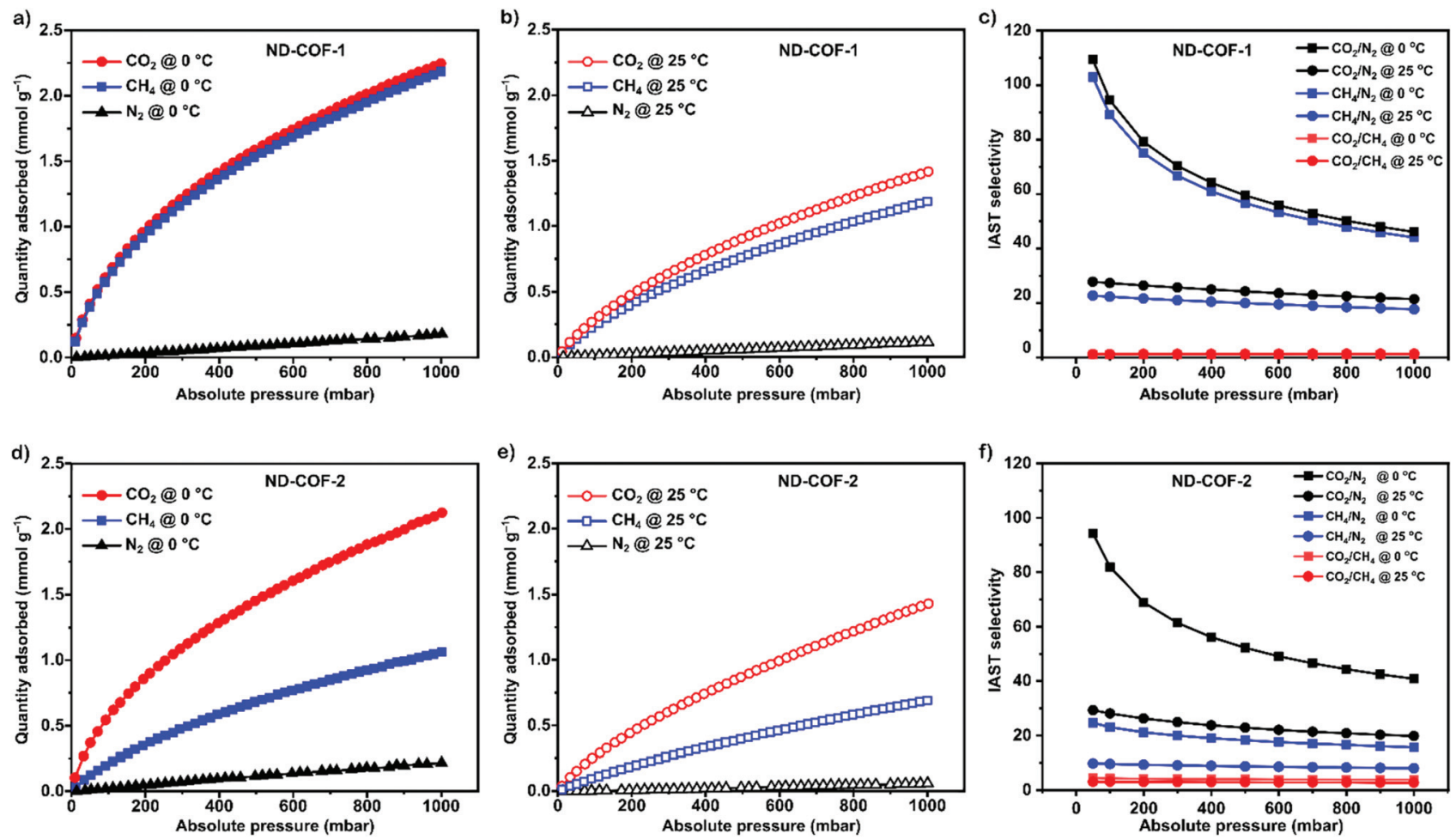

Fig. 3 Gas adsorption and selectivity data for the ND-COFs. ND-COF-1: $\mathrm{CO}_{2}, \mathrm{CH}_{4}$, and $\mathrm{N}_{2}$ adsorption isotherms at (a) $0{ }^{\circ} \mathrm{C}$ and (b) $25^{\circ} \mathrm{C}$; (c) $\mathrm{CO}_{2} /$ $\mathrm{N}_{2}, \mathrm{CH}_{4} / \mathrm{N}_{2}$, and $\mathrm{CO}_{2} / \mathrm{CH}_{4}$ ideal adsorbed solution theory (IAST) selectivity at $0{ }^{\circ} \mathrm{C}$ and $25^{\circ} \mathrm{C}$. ND-COF-2: $\mathrm{CO}_{2}, \mathrm{CH}_{4}$, and $\mathrm{N}_{2}$ adsorption isotherm at (d) $0{ }^{\circ} \mathrm{C}$ and (e) $25^{\circ} \mathrm{C}$; (f) $\mathrm{CO}_{2} / \mathrm{N}_{2}, \mathrm{CH}_{4} / \mathrm{N}_{2}$, and $\mathrm{CO}_{2} / \mathrm{CH}_{4}$ IAST selectivity at $0{ }^{\circ} \mathrm{C}$ and $25^{\circ} \mathrm{C}$. 


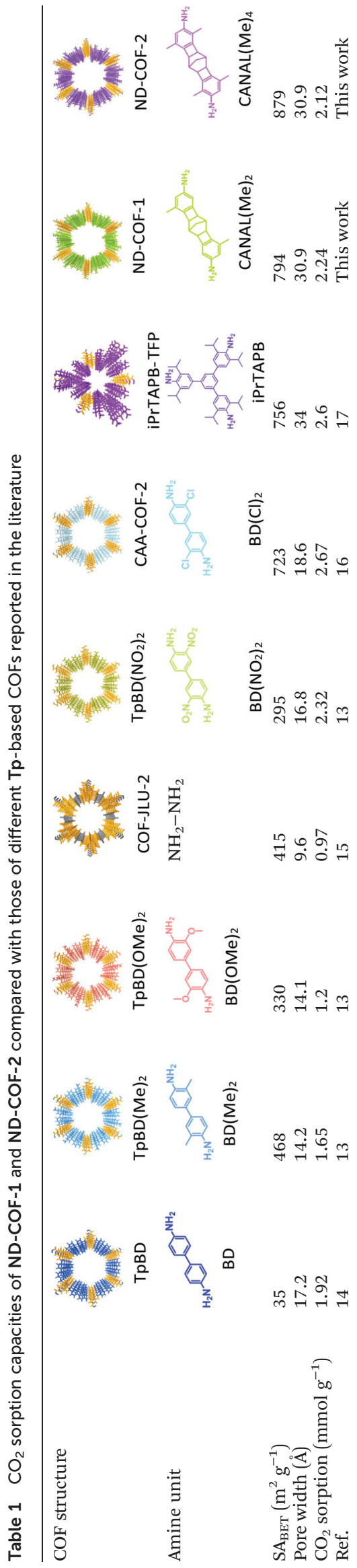

higher than those of the COFs obtained using diamines such as benzidine (TpBD; $1.92 \mathrm{mmol} \mathrm{g}^{-1}$ ), ${ }^{14}$ ortho-tolidine (TpBD $(\mathbf{M e})_{2} ; 1.65$ mmol g $\left.{ }^{-1}\right),{ }^{13}$ ortho-toluidine (TpBD(OMe $)_{2}$; $1.2 \mathrm{mmol} \mathrm{g}^{-1}$ ), ${ }^{13}$ and hydrazine units (COF-JLU2; $0.97 \mathrm{mmol}$ $\left.\mathrm{g}^{-1}\right) .{ }^{15}$ However, the $\mathrm{CO}_{2}$ sorption capacities of ND-COF-1 and ND-COF-2 were comparable to those of other COFs prepared using diamines such as dinitrobenzidine $\left(\mathbf{T p B D}\left(\mathbf{N O}_{2}\right)_{2}\right.$; $\left.2.32 \mathrm{mmol} \mathrm{g}^{-1}\right),{ }^{13} \mathrm{BD}(\mathrm{Cl})_{2}$ (CAA-COF-2; $\left.2.67 \mathrm{mmol} \mathrm{g}^{-1}\right),{ }^{16}$ and triamine (e.g., TAPB) (iPrTAPB-TFP, $\left.2.6 \mathrm{~g}^{-1}\right) .{ }^{17}$ The gas-pair selectivities of both COFs were calculated using the ideal adsorbed solution theory (IAST) at a partial equilibrium pressure of 0.5 bar/0.5 bar (Fig. $3 \mathrm{c}$ and f). ND-COF-1 and ND-COF-2 exhibited high $\mathrm{CO}_{2} / \mathrm{N}_{2}$ selectivities of 46 and 41 at $0{ }^{\circ} \mathrm{C}$ and $1 \mathrm{bar}$, respectively. These selectivities were reduced by approximately $50 \%$ upon increasing the temperature to $25{ }^{\circ} \mathrm{C}$ under atmospheric pressure. Remarkably, ND-COF-1 showed an excellent $\mathrm{CH}_{4} / \mathrm{N}_{2}$ selectivity of 43 at $0{ }^{\circ} \mathrm{C}$, which is $175 \%$ higher than that exhibited by ND-COF-2. The performance results show the potential of employing kinked norbornane-based COFs in industrial applications, exploiting their ease of preparation, high sorption capacity, and high gas-pair selectivity.

\section{Conclusions}

In summary, we have synthesized crystalline and porous $\beta$-ketoenamine-linked kinked norbornane COFs (ND-COF-1 and ND-COF-2) and investigated the effect of kinked CANALunit-decorated frameworks in gas separation. Both COFs exhibited high chemical stability in different organic polar and non-polar solvents, including acidic media. However, ND-COF-2 disintegrated in alkaline media, whereas ND-COF-1 remained stable. Interestingly, due to the presence of a methyl-functional-group-decorated pore interior, the $\mathrm{CO}_{2}$ adsorption efficiency of ND-COF-2 was approximately 100\% higher than its $\mathrm{CH}_{4}$ sorption efficiency. According to the IAST calculations, ND-COF-1 showed $175 \%$ higher $\mathrm{CH}_{4} / \mathrm{N}_{2}$ selectivity than ND-COF-2 at $0{ }^{\circ} \mathrm{C}$ and 1 bar. In future studies, new designs of CANAL-based linkers through pre-synthetic or postsynthetic functionalization will be explored to improve the porosity and selectivity of the obtained COFs, thereby enhancing their separation performance.

\section{Author contributions}

SK and MAH have contributed equally. MAH: Ligand design, investigation, validation, analysis, data curation, gas separation, and writing - original draft. SK: COFs - design, synthesis, characterization, investigation, validation, formal analysis, data curation, visualization, refinement, and writing original draft. AD: Modeling, simulation, and computational analysis. MAA: Modeling, simulation, computational analysis, and supervision. GS: Conceptualization, methodology, resources, visualization, writing - review \& editing, supervision, funding acquisition, and project administration. 


\section{Conflicts of interest}

There are no conflicts to declare.

\section{Acknowledgements}

SK and MAH thank King Abdullah University of Science and Technology (KAUST) for the postdoctoral fellowship. The research reported in this publication was supported by funding from KAUST. We acknowledge Rifan Hardian for the IAST calculations, Abdul Hamid Emwas for the solid-state ${ }^{13} \mathrm{C}$ CP-MAS NMR analysis, and Mohamed Nejib Hedhili for the XPS measurements. MAA thanks the Materials Chemistry Consortium for HPC access (EP/T022213).

\section{Notes and references}

1 M. Dincă and J. R. Long, Chem. Rev., 2020, 120, 8037-8038.

2 Y. Wang, B. S. Ghanem, Z. Ali, K. Hazazi, Y. Han and I. Pinnau, Small Struct., 2021, 2, 2100049.

3 H. W. H. Lai, S. Liu and Y. Xia, J. Polym. Sci., Part A: Polym. Chem., 2017, 55, 3075-3081.

4 M. A. Abdulhamid, H. W. H. Lai, Y. Wang, Z. Jin, Y. C. Teo, X. Ma, I. Pinnau and Y. Xia, Chem. Mater., 2019, 31, 17671774.

5 X. Ma, H. W. H. Lai, Y. Wang, A. Alhazmi, Y. Xia and I. Pinnau, ACS Macro Lett., 2020, 9, 680-685.
6 S. J. Lyle, P. J. Waller and O. M. Yaghi, Trends Chem., 2019, 1, 172-184.

7 K. Dey, S. Mohata and R. Banerjee, ACS Nano, 2021, 15, 12723-12740.

8 M. S. Lohse and T. Bein, Adv. Funct. Mater., 2018, 28, 1705553.

9 K. Geng, T. He, R. Liu, S. Dalapati, K. T. Tan, Z. Li, S. Tao, Y. Gong, Q. Jiang and D. Jiang, Chem. Rev., 2020, 120, 8814-8933.

10 Y. Wang, C. Wu, W. Sun, Q. Pan, W. Hao, H. Liu, J. Sun, Z. Li, J. Sun and Y. Zhao, Mater. Chem. Front., 2021, 5, 944949.

11 H. Li, F. Chen, X. Guan, J. Li, C. Li, B. Tang, V. Valtchev, Y. Yan, S. Qiu and Q. Fang, J. Am. Chem. Soc., 2021, 143, 2654-2659.

12 J. H. Chong, M. Sauer, B. O. Patrick and M. J. MacLachlan, Org. Lett., 2003, 5, 3823-3826.

13 S. Chandra, S. Kandambeth, B. P. Biswal, B. Lukose, S. M. Kunjir, M. Chaudhary, R. Babarao, T. Heine and R. Banerjee, J. Am. Chem. Soc., 2013, 135, 17853-17861.

14 B. P. Biswal, S. Chandra, S. Kandambeth, B. Lukose, T. Heine and R. Banerjee, J. Am. Chem. Soc., 2013, 135, 5328-5331.

15 Z. Li, Y. Zhi, X. Feng, X. Ding, Y. Zou, X. Liu and Y. Mu, Chem. - Eur. J., 2015, 21, 12079-12084.

16 D. B. Shinde, M. Ostwal, X. Wang, A. M. Hengne, Y. Liu, G. Sheng, K.-W. Huang and Z. Lai, CrystEngComm, 2018, 20, 7621-7625.

17 D. Kaleeswaran, P. Vishnoi and R. Murugavel, J. Mater. Chem. C, 2015, 3, 7159-7171. 